\title{
Hypoxia-induced upregulation of BMX kinase mediates therapeutic resistance in acute myeloid leukemia
}

\author{
Jolieke G. van Oosterwijk, ${ }^{1}$ Daelynn R. Buelow, ${ }^{2}$ Christina D. Drenberg, ${ }^{2}$ Aksana Vasilyeva, ${ }^{1}$ Lie Li, ${ }^{1}$ Lei Shi, ${ }^{3}$ Yong-Dong Wang, ${ }^{4}$ \\ David Finkelstein, ${ }^{4}$ Sheila A. Shurtleff, ${ }^{5}$ Laura J. Janke, ${ }^{5}$ Stanley Pounds, ${ }^{3}$ Jeffrey E. Rubnitz, ${ }^{6}$ Hiroto Inaba, ${ }^{6}$ Navjotsingh Pabla, ${ }^{2}$ \\ and Sharyn D. Baker ${ }^{2}$ \\ 'Department of Pharmaceutical Sciences, St. Jude Children's Research Hospital, Memphis, Tennessee, USA. ²Division of Pharmaceutics, College of Pharmacy and Comprehensive Cancer Center, The Ohio \\ State University, Columbus, Ohio, USA. ${ }^{3}$ Department of Biostatistics, ${ }^{4}$ Department of Computational Biology, ${ }^{5}$ Department of Pathology, and ${ }^{6}$ Department of Oncology, St. Jude Children's Research Hospital, \\ Memphis, Tennessee, USA.
}

\begin{abstract}
Oncogenic addiction to the Fms-like tyrosine kinase 3 (FLT3) is a hallmark of acute myeloid leukemia (AML) that harbors the FLT3-internal tandem duplication (FLT3-ITD) mutation. While FLT3 inhibitors like sorafenib show initial therapeutic efficacy, resistance rapidly develops through mechanisms that are incompletely understood. Here, we used RNA-Seqbased analysis of patient leukemic cells and found that upregulation of the Tec family kinase BMX occurs during sorafenib resistance. This upregulation was recapitulated in an in vivo murine FLT3-ITD-positive (FLT3-ITD+) model of sorafenib resistance. Mechanistically, the antiangiogenic effects of sorafenib led to increased bone marrow hypoxia, which contributed to HIF-dependent BMX upregulation. In in vitro experiments, hypoxia-dependent BMX upregulation was observed in both AML and non-AML cell lines. Functional studies in human FLT3-ITD+ cell lines showed that BMX is part of a compensatory signaling mechanism that promotes AML cell survival during FLT3 inhibition. Taken together, our results demonstrate that hypoxia-dependent upregulation of BMX contributes to therapeutic resistance through a compensatory prosurvival signaling mechanism. These results also reveal the role of off-target drug effects on tumor microenvironment and development of acquired drug resistance. We propose that the bone marrow niche can be altered by anticancer therapeutics, resulting in drug resistance through cell-nonautonomous microenvironment-dependent effects.
\end{abstract}

\section{Introduction}

Advances in genomics, as well as the development of molecularly targeted drugs, have transformed cancer therapy (1). Identification of genomic alterations and oncogenic addictions in specific cancer subtypes has provided us with increased ability to predict drug responsiveness to targeted therapeutics (1). Nevertheless, drug resistance generally develops in tumors that are initially sensitive to single-agent treatment (2). Multiple mechanisms contribute to acquired drug resistance, including development of secondary mutations or increased target gene expression, reduced cellular drug accumulation, activation of compensatory signaling pathways, alterations in the tumor microenvironment, and clonal selection of resistant cell subpopulations (2). Identifying and overcoming resistance mechanisms is currently a major challenge in cancer therapeutics.

Acute myeloid leukemia (AML) is a clear example where targeted therapies have still not led to significant clinical benefits, in part because of drug resistance as well as molecular heterogeneity (3). Several genes are frequently mutated, rearranged, or deregulated in AML cells. Among these, activating internal tandem duplication (ITD) mutations in Fms-like tyro-

Conflict of interest: The authors have declared that no conflict of interest exists Submitted: November 21, 2016; Accepted: November 2, 2017

Reference information: / Clin Invest. 2018;128(1):369-380.

https://doi.org/10.1172/JCI91893. sine kinase 3 (FLT3) are detected in about a quarter of AML patients (3). These FLT3-ITD-harboring (FLT3-ITD ${ }^{+}$) AML cells are dependent on FLT3 signaling for survival and present an attractive therapeutic target (4). As a result, multiple tyrosine kinase inhibitors (TKIs) that inhibit FLT3 have been clinically evaluated. These studies have shown that FLT3 inhibitors like sorafenib are effective initially, but drug resistance develops rapidly (4). Mutations within the FLT3 tyrosine kinase domain (TKD) contribute to TKI resistance (5), but other mechanisms have remained relatively unexplored.

Like most clinically approved TKIs (6), sorafenib has multiple targets, and these "off-target" activities contribute to both efficacy and toxicities (7). While its efficacy in renal and hepatocellular cancer is attributed to VEGFR2 and PDGFR inhibition (7), FLT3 is likely the major target in FLT3-ITD ${ }^{+}$AML (8). Moreover, TGF- $\beta$-activated protein kinase 1 (TAK1) inhibition in keratinocytes is responsible for skin toxicities associated with sorafenib treatment (9). Here we show that TKI-mediated multiple-target inhibition can also contribute to drug resistance. We propose a unique resistance mechanism wherein sorafenib's antiangiogenic activity leads to bone marrow hypoxia, a therapy-induced alteration in the microenvironment that contributes to the development of adaptive drug resistance in AML cells. Importantly, we have identified the Tec kinase BMX as a hypoxia-inducible gene, which imparts sorafenib resistance by activating prosurvival signaling pathways in AML cells. 
A

ChrX 15,513,900

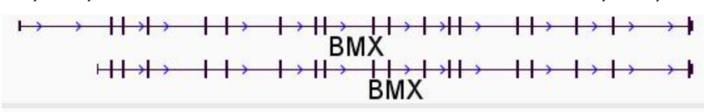

P1

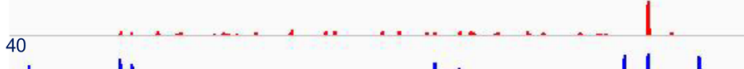

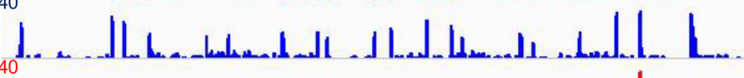

P2

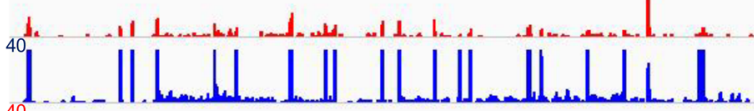

P3

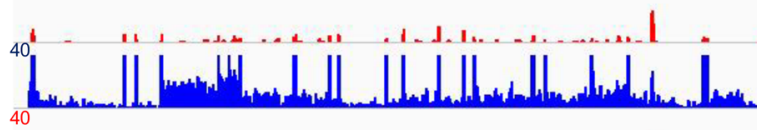

P4

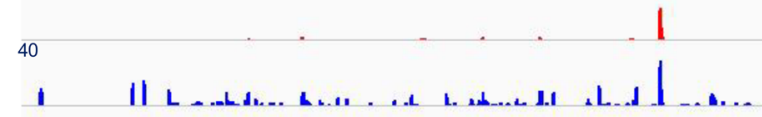

B

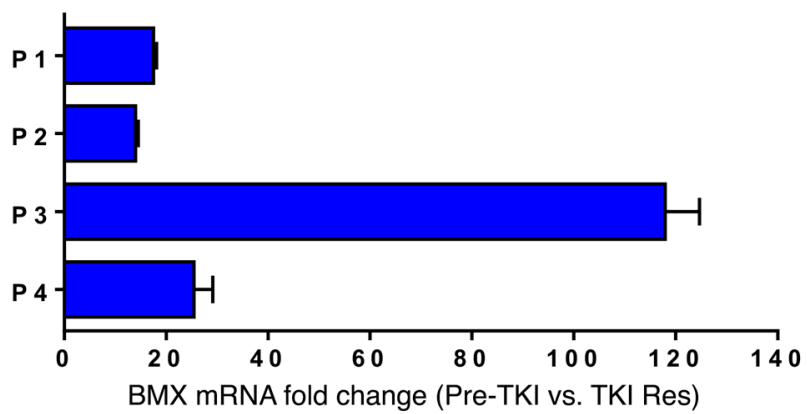

C

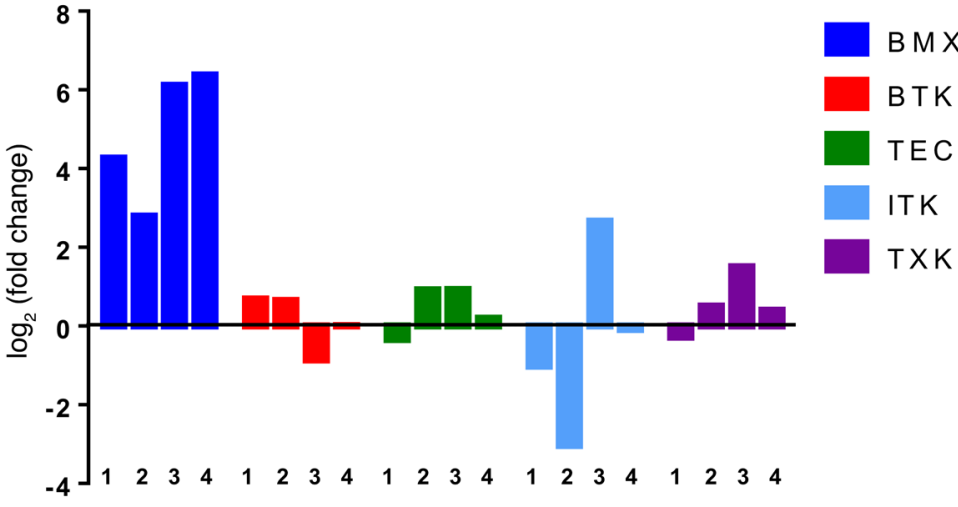

Figure 1. Transcriptional upregulation of $B M X$ in AML patients during sorafenib resistance. RNA-Seq analysis of bone marrow aspirates from 4 patients collected at initial relapse of AML (Pre-TKI) and at development of resistance to TKI therapy (TKI Res). (A) Integrative Genomics Viewer snapshot of RNA-Seq data showing genomic locus of 2 BMX transcripts. (B) BMX overexpression was confirmed using RT-PCR (in triplicate). (C) Exon junction read counts from RNA-Seq analysis for each patient representing log fold change of resistance minus diagnosis; $x$ axis shows patients 1-4 per Tec kinase.

\section{Results}

The nonreceptor tyrosine kinase BMX is upregulated in FLT3-ITD $A M L$ patients during the emergence of sorafenib resistance. In FLT3-ITD ${ }^{+}$AML, the emergence of secondary kinase domain (KD) mutations is one resistance mechanism that develops in response to sorafenib treatment (5). However, drug resistance can also develop by nonmutational (adaptive) mechanisms through alterations in transcription and/or protein signaling
Pre-TKI

TKI Res

Pre-TKI

TKI Res

Pre-TKI

TKI Res

Pre-TKI

TKI Res

contributing to the maintenance of prosurvival signaling essential for tumor maintenance and growth (10). To identify such previously unknown additional mechanisms of sorafenib resistance, we performed whole transcriptome sequencing (RNASeq) on bone marrow aspirates from 4 patients with FLT3-ITD $^{+}$AML before TKI treatment and after the emergence of sorafenib resistance. Notably, these patients also developed FLT3 KD mutations at TKI resistance; $33 \%-41 \%$ of the total blast population harbored $\mathrm{KD}$ mutations, suggesting that additional resistance mechanisms may be involved in blast samples not harboring KD mutations (Supplemental Table 1; supplemental material available online with this article; https://doi.org/10.1172/ JCI91893DS1). The change in FLT3-ITD allelic ratio, which describes the number of ITD-mutated alleles compared with the number of WT alleles, and the change in the number of unique ITD sequence from before treatment to sorafenib resistance are also summarized in Supplemental Table 1. Comparative analysis of RNA-Seq data showed that BMX (bone marrow tyrosine kinase gene in chromosome $\mathrm{X}$ ) expression was upregulated in all the 4 patients during sorafenib resistance (Supplemental Tables 2 and 3; and Figure 1A). Increased BMX expression was further confirmed by quantitative PCR (qPCR) analysis (Figure 1B). BMX is a nonreceptor tyrosine kinase belonging to the Tec kinase family, which is highly expressed in myeloid and endothelial cells $(11,12)$. BMX contains an SH2 domain that binds to tyrosine-phosphorylated proteins and a $\mathrm{PH}$-like domain, which mediates membrane targeting by binding to phosphatidylinositol 3,4,5-triphosphate (PIP3) (13). BMX regulates several signal transduction pathways and has been implicated not only in proliferation and survival of various cancer cell types, but also in imparting resistance to chemotherapeutics (14). We next explored whether other Tec family members are upregulated during sorafenib resistance since BTK has been implicated as a therapeutic target in AML (15). We found that only BMX was strongly upregulated, while other Tec kinases, including BTK, were not increased (Figure 1C). Moreover, based on exome sequencing and SNP6 array analysis, the $B M X$ gene did not show any mutations or copy number changes, indicating that BMX upregulation during sorafenib resistance is not likely due to copy number changes.

Sorafenib induces BMX upregulation in a MOLM13 FLT3-ITD mouse model. To decipher the molecular mechanisms that contribute to BMX upregulation during sorafenib resistance, we used a MOLM13 FLT3-ITD ${ }^{+}$mouse model of sorafenib resistance. To understand the contribution of FLT3 inhibition to BMX upregulation, we also included crenolanib, another FLT3 inhibitor (16). In a pilot survival study, mice bearing MOLM13 FLT3-ITD ${ }^{+}$ cells were treated with vehicle, crenolanib, or sorafenib until the 
A

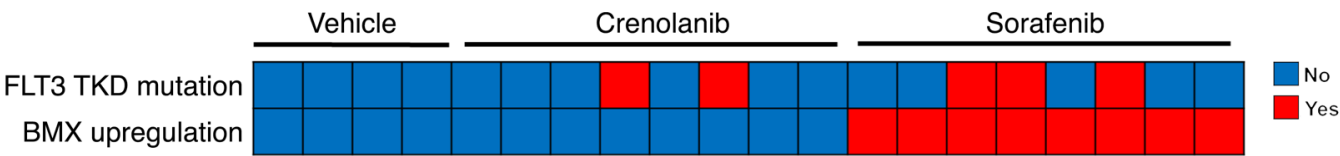

B

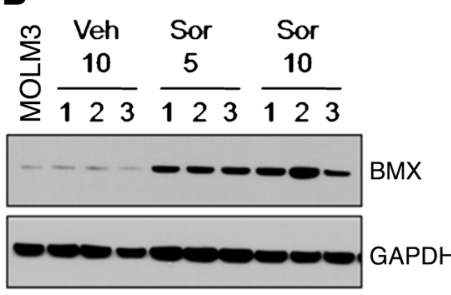

c

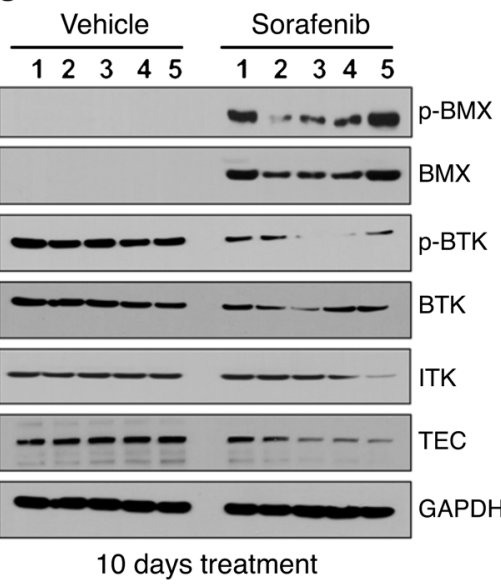

D

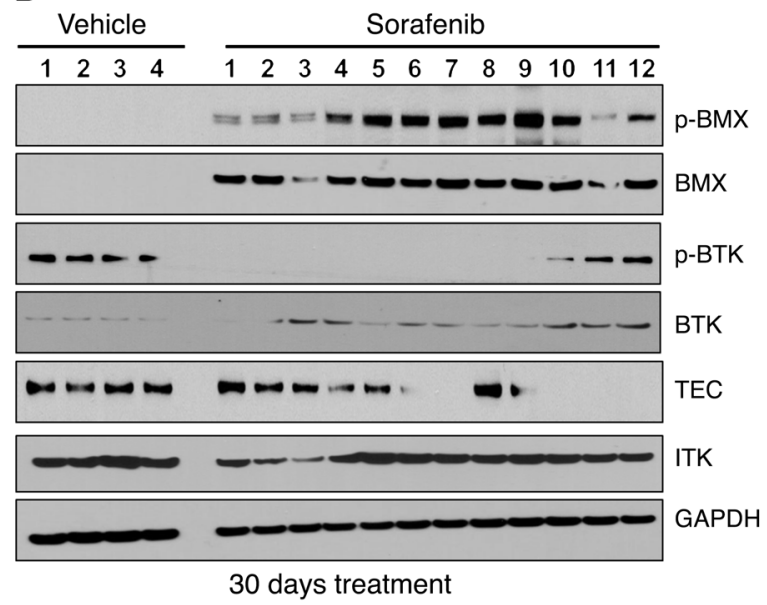

$\mathbf{E}$

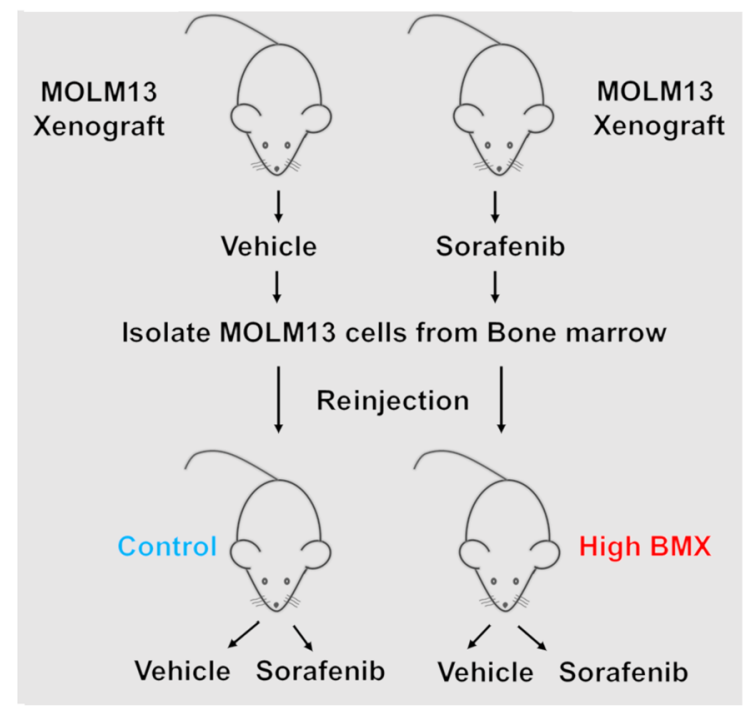

$\mathbf{F}$

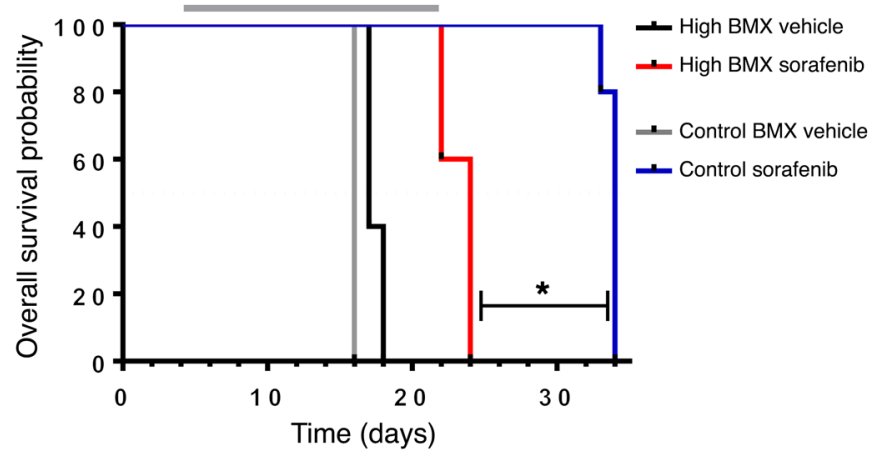

Figure 2. Sorafenib induces BMX upregulation in a MOLM13 FLT3-ITD+ xenograft model of sorafenib resistance. (A) MOLM13 cells were administered to NSC mice by tail vein injection (TVI), and 10 days after TVI mice were treated with vehicle, crenolanib, or sorafenib until the time of leukemic progression (on days 17 , 24, and 40 after TVI, respectively), at which time bone marrow MOLM13 cells were isolated, FLT3 tyrosine kinase domain (TKD) mutation status was assessed by deep amplicon sequencing, and BMX protein expression was assessed by Western blotting. A schematic representation of FLT3 TKD mutation status and BMX upregulation is shown; each box represents an individual sample. (B) NSG mice engrafted with MOLM13 cells for 10 days were treated with vehicle or sorafenib for 5 or 10 days. BMX upregulation was observed after 5 days of sorafenib treatment. (C and $\mathbf{D})$ After 10 (C) or 30 days (D) of sorafenib treatment, BMX is the only Tec kinase that is activated by sorafenib in comparison with vehicle-treated mice as shown by increased phospho-BMX ( $p$-BMX); $p$-BTK and Tec kinase are downregulated. (B-D) Western blots are from 1 experiment. (E and $\mathbf{F}$ ) MOLM13 cells were obtained from the bone marrow of mice treated with vehicle or sorafenib at the time of leukemic progression (day 14 after TVI for vehicle; day 40 after TVI for sorafenib). Expression of BMX and $p$-BMX was determined by Western blot, cells with low/absent (D, lanes $1-4)$ or high p-BMX expression (D, lanes 2, 5-10, 12) were pooled, and $1 \times 10^{6}$ cells were administered i.v. to NSG mice. Starting 7 days after TVI, mice were treated with vehicle $(n=5)$ or sorafenib $(n=5)$ for 18 days. Survival was significantly longer in mice injected with MOLM13 cells with low versus high BMX expression (34 vs. 24 days; ${ }^{*} P=0.0031$, Kaplan-Meier analysis).

development of resistance. Emerging resistance was determined by an increase in leukemic cell outgrowth determined from bioluminescence imaging (Supplemental Figure 1A). Mice treated with vehicle, crenolanib, or sorafenib survived a median of 16 days, 28 days, and 45 days, respectively (Supplemental Figure 1B). In a follow-up study, mice were given the same treatments, bone marrow was harvested on days 17, 24, and 40 in vehicle-, crenolanib-, and sorafenib-treated mice, respectively, BMX expression was determined by Western blotting, and FLT3 TKD mutations were assessed by deep amplicon sequencing. Interestingly, BMX expression was not observed in mice treated with vehicle or crenolanib, while BMX was significantly upregulated 
A

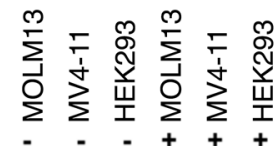

Hypoxia:

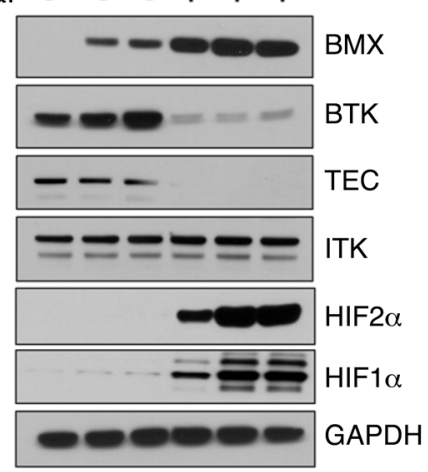

C

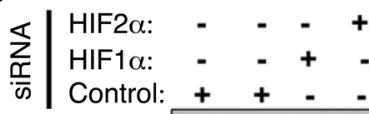

Hypoxia:

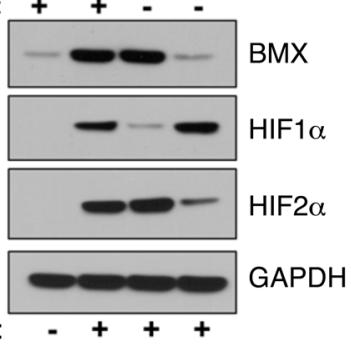

E

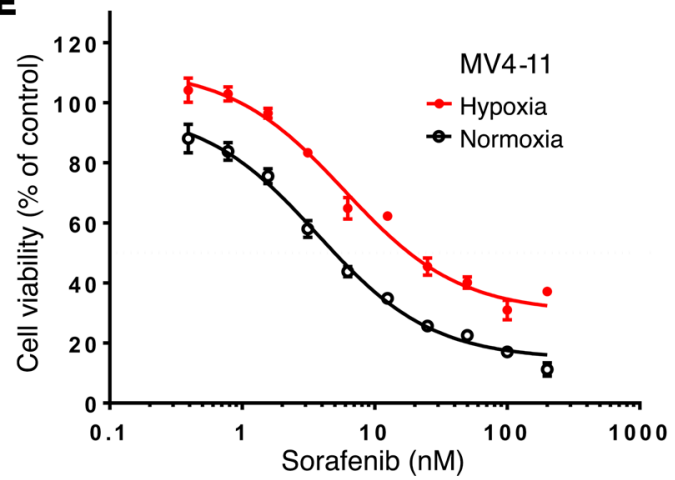

$\mathbf{F}$

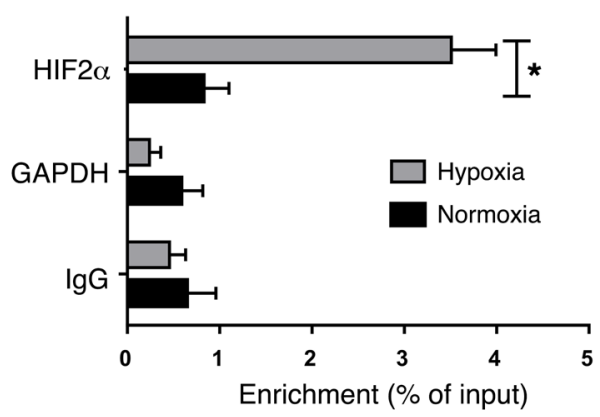

B

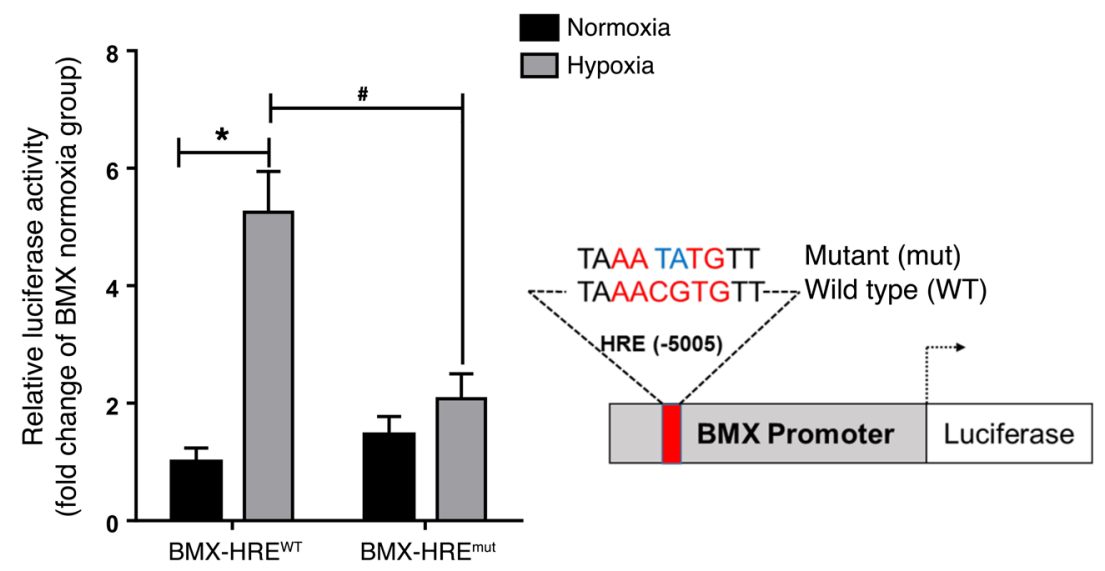

D

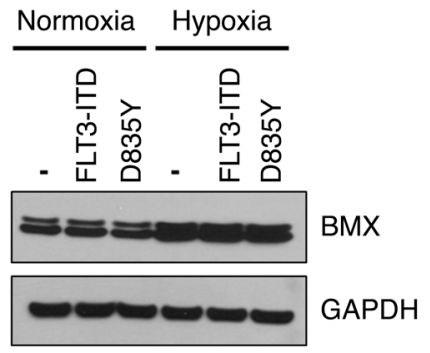

G

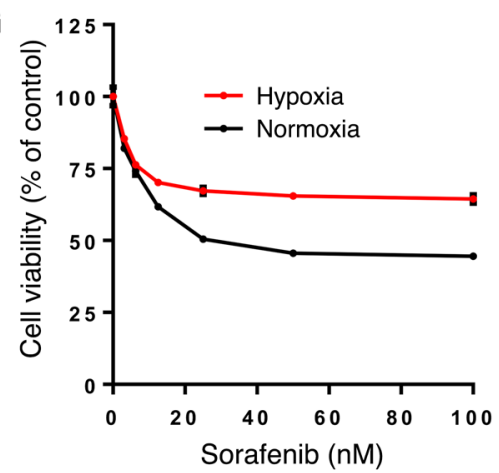

MOLM13
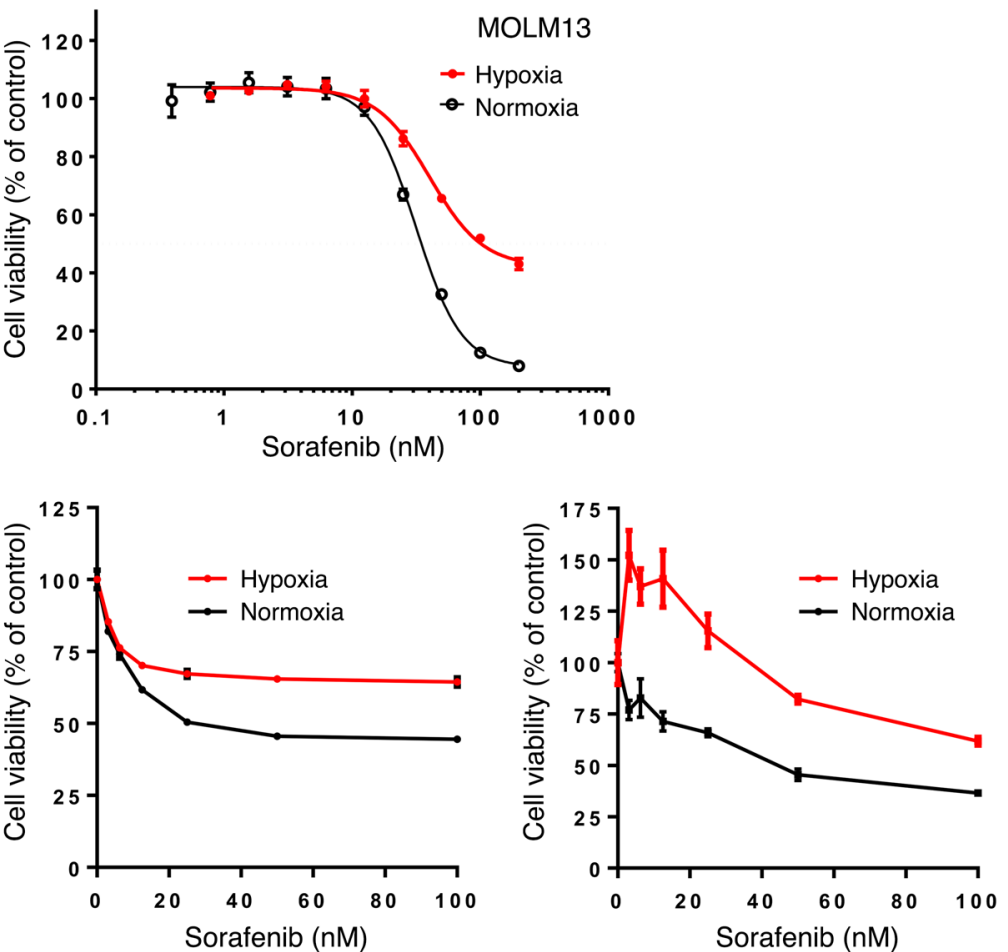
Figure 3. In vitro upregulation of BMX under hypoxic conditions. (A) Indicated cell lines were cultured in normoxia or 1\% hypoxia for 24 hours followed by Western blot analysis of indicated proteins. Representative blots from at least 3 independent experiments. (B) The right panel schematically represents the BMX promoter near the HRE site and the mutant construct. HEK293 cells were transfected with an empty promoter, BMX promoter, or the BMX promoter with mutated HRE element. After 1 day, cells were transferred to a hypoxia chamber for 24 hours. Hypoxic conditions upregulated $\mathrm{BMX}$ transcription ( ${ }^{*} P=0.017$, Welch's $t$ test), which was abrogated by the deletion of the HRE element ( $P=0.025$, Welch's $t$ test). Data shown as mean of 3 replicates and representative of 2 independent experiments. (C) HEK293 cells were transfected with control, HIF1 $\alpha$, or HIF2 $\alpha$ siRNA, followed by hypoxia treatment and Western blot analysis. HIF2 $\alpha$ knockdown abrogated hypoxia-mediated BMX upregulation. Representative blots from at least 2 independent experiments. (D) BMX expression was determined in Baf3 cells expressing indicated FLT3 constructs after cell culture in normoxic or hypoxic ( 24 hours) conditions. Representative blots from at least 2 independent experiments. (E) MOLM13 and MV4-11 cells were grown under hypoxic and normoxic conditions (24 hours) followed by sorafenib treatment, and cell viability was assessed by MTT assay. Representative of 3 independent experiments (18 replicates). (F) ChIP assay in MOLM13 cells showed that HIF2 $\alpha$ can bind the BMX promoter under hypoxic conditions ( ${ }^{*} P=0.014$, Welch's $t$ test). Data shown as mean of 3 replicates and representative of 3 independent experiments. (G) Diagnostic FLT3-ITD+ blast samples from patient A (left) and patient B (right) were treated with sorafenib under normoxic or hypoxic conditions, and cell viability was assessed by CellTiter Glo (1 experiment, 3 replicates). These results indicate that hypoxia can cause sorafenib resistance in patientderived primary AML cells.

in the sorafenib-treated group (Supplemental Figure 1B). Analysis of FLT3-ITD TKD mutation status showed that 2 of 8 crenolanib-treated mice and 3 of 8 sorafenib-treated mice developed TKD mutations (Figure 2A and Supplemental Table 4). These results indicated that BMX upregulation is likely to be independent of the presence of TKD mutations and not a direct effect of FLT3 inhibition, since the crenolanib-treated group did not show any BMX upregulation. To further confirm the independence of BMX upregulation from the presence of TKD mutations, we performed a short-term experiment of 5 and 10 days of sorafenib treatment, when neither an outgrowth of leukemia cells nor sorafenib resistance is observed (Supplemental Figure 1A), and found that BMX expression was already increased after 5 and 10 days of sorafenib treatment as compared with the vehicle-treated group (Figure 2B). Next, we generated a phospho-BMX antibody against the autophosphorylation site of BMX (Supplemental Figure 2), which could be used as a readout of BMX kinase activity. Indeed, we found that phospho-BMX levels were elevated in bone marrow leukemic cells from sorafenib-treated mice (Figure 2C and Supplemental Figure 3A). Protein levels of other Tec kinases, including BTK, were not increased compared with samples from vehicle-treated mice. These results obtained at early time points were further confirmed in samples obtained from mice treated with sorafenib for 30 days, at the time of leukemic outgrowth (Figure 2D and Supplemental Figure 3B). Furthermore, we carried out $\mathrm{BMX}$ in vitro kinase assay, which showed that BMX kinase activity was elevated in the AML cells derived from sorafenib-treated mice as compared with vehicle-treated groups (Supplemental Figure 3C). To determine whether high BMX expression contributes to sorafenib resistance, bone marrow MOLM13 cells with low/absent and high phospho-BMX expression generated in vivo, from vehicle- and sorafenib-treated mice, respectively, were pooled and readministered i.v., and mice were then treated with vehicle or sorafenib (Figure 2E). Although median survival was similar in vehicle-treated mice injected with cells with low/absent versus high BMX (16 vs. 17 days), survival was significantly longer in mice injected with MOLM13 cells with low BMX expression versus high BMX expression (34 vs. 24 days; $P=0.0031$ ) (Figure $2 \mathrm{~F})$. Thus, we could experimentally mimic the BMX upregulation observed in sorafenib-resistant patients in our in vivo model.

$B M X$ is a hypoxia-inducible gene. Since we found that BMX upregulation was likely FLT3-independent, we next examined the direct effect of sorafenib treatment on BMX expression in cultured cells in the presence or absence of mesenchymal stromal cells (MSCs), which can contribute to drug resistance (17). We did not observe any effect of sorafenib treatment on BMX expression in vitro in MOLM13 cells in the presence or absence of MSCs (Supplemental Figure 4). Interestingly, BMX upregulation could be hypoxia-dependent, since a previous study has shown that BMX expression can be induced by ischemia in endothelial cells (18). Also, while both crenolanib and sorafenib are FLT3 inhibitors, sorafenib also inhibits VEGFR2 (6), which has an antiangiogenic effect. We therefore hypothesized that the antiangiogenic activity of sorafenib caused hypoxia in the bone marrow, which might contribute to hypoxia-dependent BMX upregulation in AML cells. To test this hypothesis, we initially treated AML cell lines along with HEK293 cells with 1\% hypoxia and found a significant increase in BMX expression in all the cell lines (Figure 3A); hypoxia treatment did not lead to increased expression of other Tec kinases. These results indicate that BMX may be a hypoxia-regulated gene in multiple cell types. Indeed, BMX expression has been reported to be elevated in clear-cell renal cell carcinomas (19), which generally have von Hippel-Lindau (VHL) mutations and increased expression of hypoxia-inducible factors (HIFs). Analysis of RNA-Seq data from The Cancer Genome Atlas (TCGA) as well as examination of BMX protein expression in renal cell carcinoma cell lines corroborated these findings (Supplemental Figure 5). Gene-set differential expression analysis of patient samples at TKI resistance compared with pre-TKI samples showed alterations in hypoxia-related pathways and upregulation of hypoxia response genes such as ANGPTL4 (ref. 20 and Supplemental Table 5). We also found a hypoxia-responsive element (HRE) in the BMX promoter (Figure 3B), and a luciferase reporter assay showed HRE-dependent promoter activity under hypoxic conditions (Figure 3B). Next, we used RNAi-mediated HIF1 $\alpha$ and HIF $2 \alpha$ knockdown in HEK293 cells and found that HIF $2 \alpha$ knockdown reduced BMX upregulation observed under hypoxic conditions (Figure 3C). These results were validated by use of a dissimilar HIF $2 \alpha$ siRNA (Supplemental Figure 6). Importantly, the murine samples that showed BMX upregulation during sorafenib resistance (Figure 2, C and D) also showed elevated HIF2 $\alpha$ expression (Supplemental Figure 7, A-D), and immunohistochemical studies showed that sorafenib-treated mice have increased expression of both BMX and HIF $2 \alpha$ in the leukemic cells in the bone marrow (Supplemental Figure 7E). While BMX upregulation was found to be HIF-dependent, we next tested FLT3 mutation dependence. In Baf3 cells, BMX upregulation under hypoxic conditions was found to be independent of FLT3ITD or FLT3 TKD mutation status (Figure 3D). Along with BMX 
A

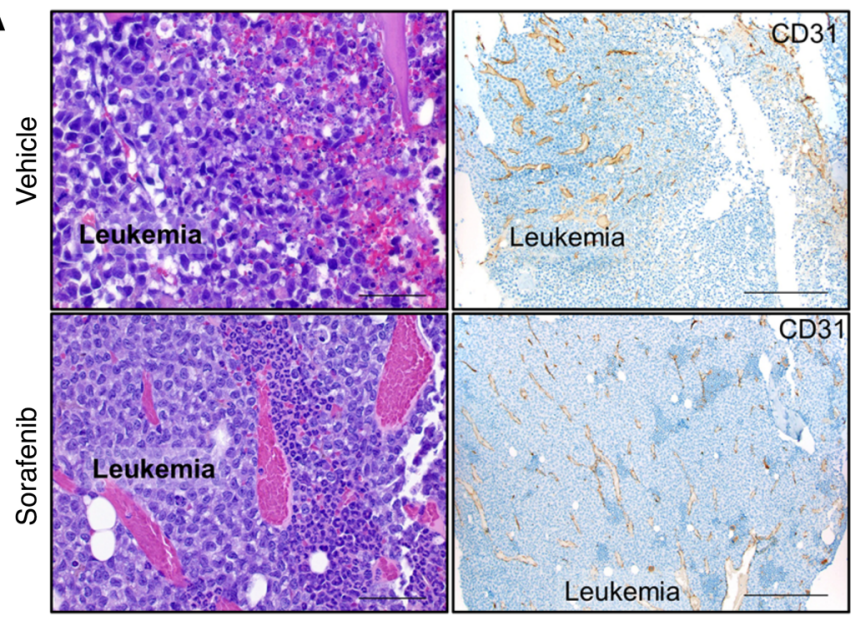

B

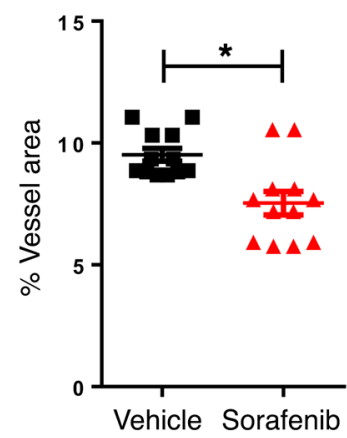

C

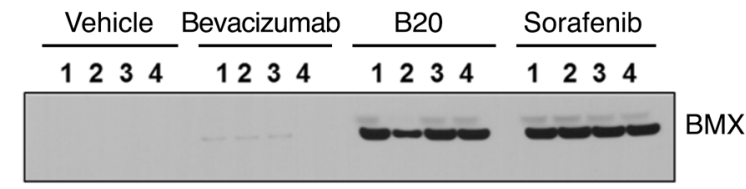

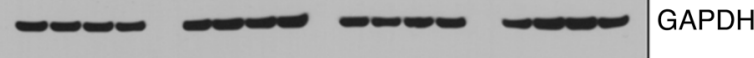

Figure 4. VEGF inhibition is sufficient to induce BMX expression in a MOLM13 FLT3-ITD+ xenograft model. Mice engrafted with MOLM13 cells were treated with vehicle or sorafenib for 5 days, after which the hind limbs were embedded in paraffin for assessment of percentage vessel area over total bone marrow area using CD31 staining. (A) Representative images of bone marrow histology and CD31 staining (original magnification, $\times 40$ ) from mice treated with vehicle $(n=12)$ or sorafenib $(n=12)$. More than 3 fields were analyzed for each sample per mouse. Scale bar: $50 \mu \mathrm{M}$. (B) The decrease in vessel area in mice treated with sorafenib indicates an increase in hypoxia ( ${ }^{*} P=0.002$, Welch's $t$ test). (C) Starting on day 5 after injection of MOLM13 FLT3-ITD+ cells, NSG mice were treated with vehicle, sorafenib, bevacizumab, or B20. On day 10 of sorafenib treatment and day 13 of bevacizumab or B20 treatment, Western blot analysis of bone marrow showed that B20-mediated VEGF inhibition was sufficient to induce BMX upregulation in MOLM13 cells. Western blots are from 1 experiment.

upregulation, hypoxia also led to sorafenib resistance in MV4-11 and MOLM13 cell lines (Figure 3E). Chromatin immunoprecipitation studies in MOLM13 cells further confirmed that HIF $2 \alpha$ can potentially bind the BMX promoter under hypoxic conditions (Figure 3F). Finally, we used patient-derived FLT3-ITD ${ }^{+}$primary AML blast samples from 2 patients and found that hypoxia can cause sorafenib resistance in primary cancer cells (Figure 3G).

Endothelial VEGF inhibition is sufficient to induce BMX upregulation in AML xenograft models. While these in vitro experiments suggested that BMX upregulation is hypoxia-dependent, in vivo experiments were carried out to examine this further. First, we tested whether sorafenib treatment had antiangiogenic effects in the bone marrow. To this end, NSG mice were injected with MOLM13 FLT3-ITD $^{+}$cells, and 10 days later, mice were treated with sorafenib for 5 days. After only 5 days of sorafenib treatment, the total vessel area in the bone marrow, as indicated by CD31 staining, was significantly reduced (Figure 4, A and B). These results were not unexpected given the known antiangiogenic effects of sorafenib $(21,22)$. However, to directly test our hypothesis, that endothelial VEGF inhibition is sufficient to increase BMX expression in AML cells, we treated MOLM13 cell-bearing NSG mice with 2 VEGF antibodies: bevacizumab, which inhibits tumor-derived human VEGF, and B20, which inhibits murine VEGF. These experiments showed that B20-mediated endothelial VEGF inhibition was sufficient to induce BMX expression in MOLM13 AML cells obtained from the bone marrow of treated mice (Figure $4 \mathrm{C}$ ). Although we cannot completely rule out other mechanisms, these studies provide strong evidence that antiangiogenic effects of sorafenib cause bone marrow hypoxia, and contribute to BMX upregulation in AML cells.
BMX mediates sorafenib resistance in vitro and in vivo. To investigate the functional role of BMX in mediating sorafenib resistance, we overexpressed different BMX constructs in HEK293 cells and then treated these cells with sorafenib (Figure $5 \mathrm{~A})$. We found that the constitutively active BMX conferred resistance, while the dominant-negative BMX imparted sensitivity to sorafenib in HEK293 cells (Figure 5B). Parallel results were obtained in MOLM13 cells, with BMX overexpression imparting sorafenib resistance (Figure $5 \mathrm{C}$ ). As an alternate approach, shRNA-mediated BMX knockdown was done in MV4-11 cells. Along with control shRNA, 2 BMX-targeting shRNAs were used, among which shRNA-B reduced BMX expression, whereas shRNA-D was ineffective (Figure 5D). Cell viability assays showed that cells with reduced BMX expression (shRNA-B) were sensitized to sorafenib (Figure 5D). These results were validated by use of dissimilar BMX shRNA constructs (Supplemental Figure 8). Moreover, we found that hypoxia-mediated sorafenib resistance can be significantly ameliorated by BMX knockdown (Supplemental Figure 8). A comparison of endogenous BMX expression in HEK293 cells, MV4-11 cells, and MOLM13 cells under normoxia and hypoxia conditions, as well as BMX expression in cells with overexpression and knockdown of BMX, is shown in Supplemental Figure 8B. These results raised the possibility that BMX inhibition could prevent or delay the emergence of sorafenib resistance in vivo. We initially confirmed that ibrutinib, which is a potent inhibitor of Tec family kinases, including BMX, was able to reverse the sorafenib resistance in constitutively active BMX-overexpressing cells (Supplemental Figure 9). Further, NSG mice were injected with MOLM13 cells, and sorafenib treat- 
A

\begin{tabular}{l} 
A $\begin{array}{l}\text { TH } \\
\text { PH }\end{array}$ TH3 \\
\hline
\end{tabular}

B

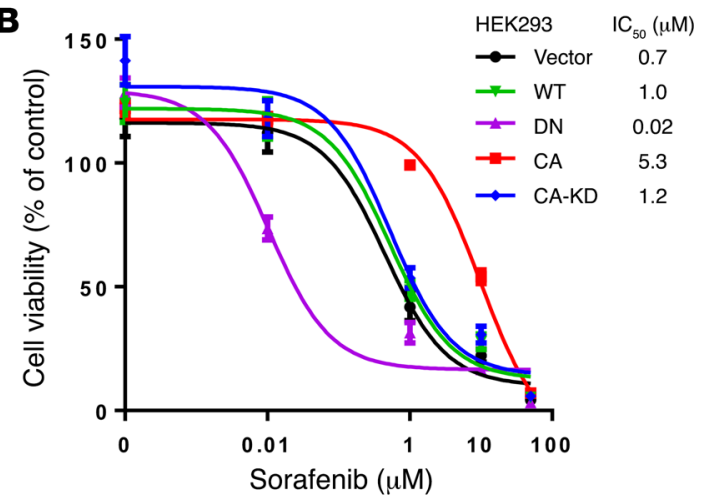

C

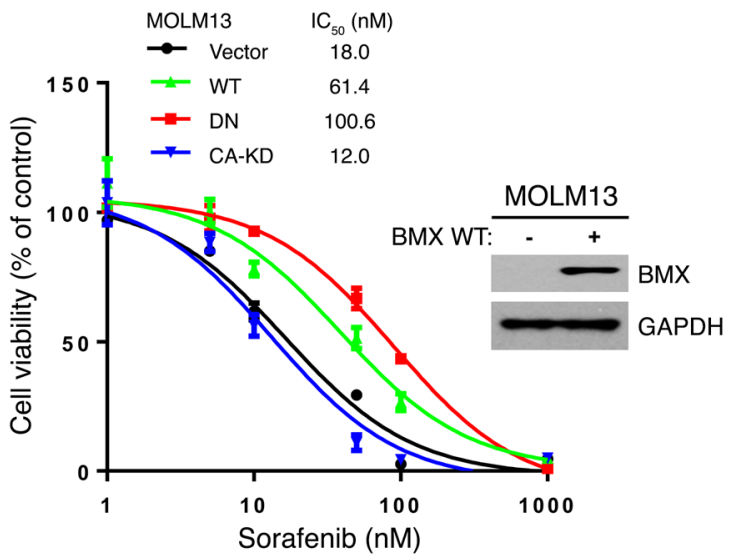

D

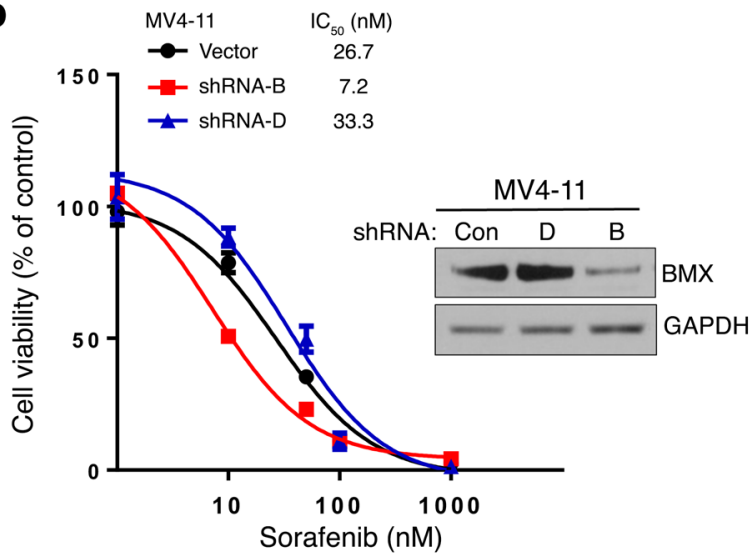

E

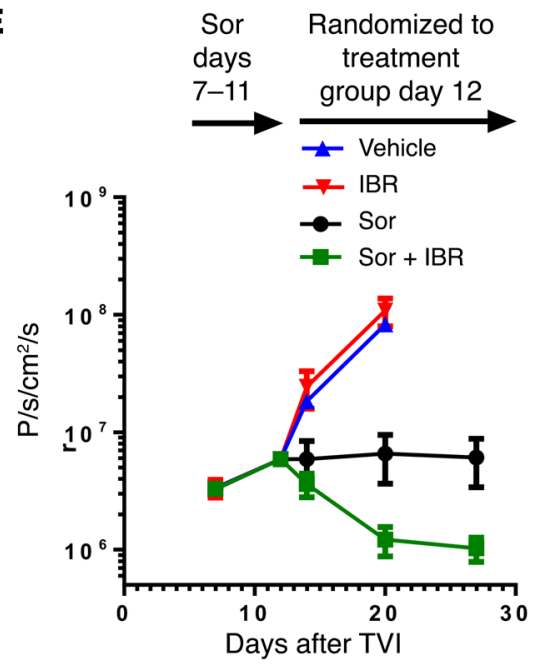

$\mathbf{F}$ Vector: Active BMX:
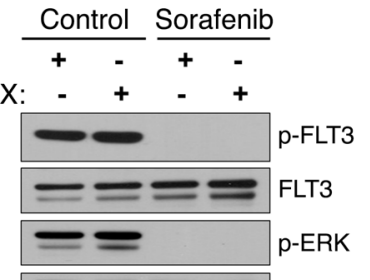
$==\equiv$ ERK $_{\text {ERK }}$ $\longrightarrow$ p-STAT5 $\longrightarrow$ STAT5 $-\ldots$ p-AKT $--\ldots$ AKT $\longrightarrow$ GAPDH
G $\begin{array}{lcl} & \text { Control } & \text { Sorafenib } \\ \text { Vector: } & + & + \\ \text { BMX shRNA: }- & + & -\end{array}$

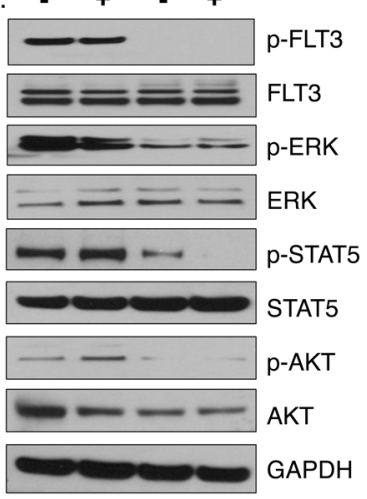

Figure 5. Functional role of BMX upregulation during sorafenib treatment. (A) Schematic representation of BMX constructs. (B-D) In an MTT cell viability assay, introduction of constitutively active BMX confers resistance to sorafenib in HEK293 (B) and MOLM13 cells (C), and BMX knockdown results in increased sensitivity to sorafenib treatment in MV4-11 cells (D). Data in B-D are from a polyclonal pool of cells, and the representative graphs are from at least 2 independently repeated experiments with 4 replicates per group. (E) Ibrutinib sensitizes leukemic cells to sorafenib in a MOLM13 FLT3-ITD+ xenograft model in vivo ( $n=5$ per treatment group). (F) Constitutively active BMX maintains STAT5 and AKT activation during FLT3 inhibition by sorafenib in MOLM13 cells. Representative blots from 3 independent experiments. (G) BMX knockdown (shRNA-B) enhanced STAT5 inhibition during sorafenib treatment in MV4-11 cells. Representative blots from 2 independent experiments.

ment was initiated for 5 days. As we have shown that BMX upregulation already occurs after 5 days of sorafenib treatment (Figure 2B), mice were then divided into 4 different groups. One group received vehicle, and one continued on sorafenib treatment. The third group received ibrutinib, and the last group received a com- bination therapy of sorafenib with ibrutinib. Daily treatment of sorafenib and ibrutinib was well tolerated, and a pharmacokinetic study showed that coadministration of both drugs did not alter plasma concentrations compared with single-agent treatment (Supplemental Figure 10). As soon as the combination treatment 

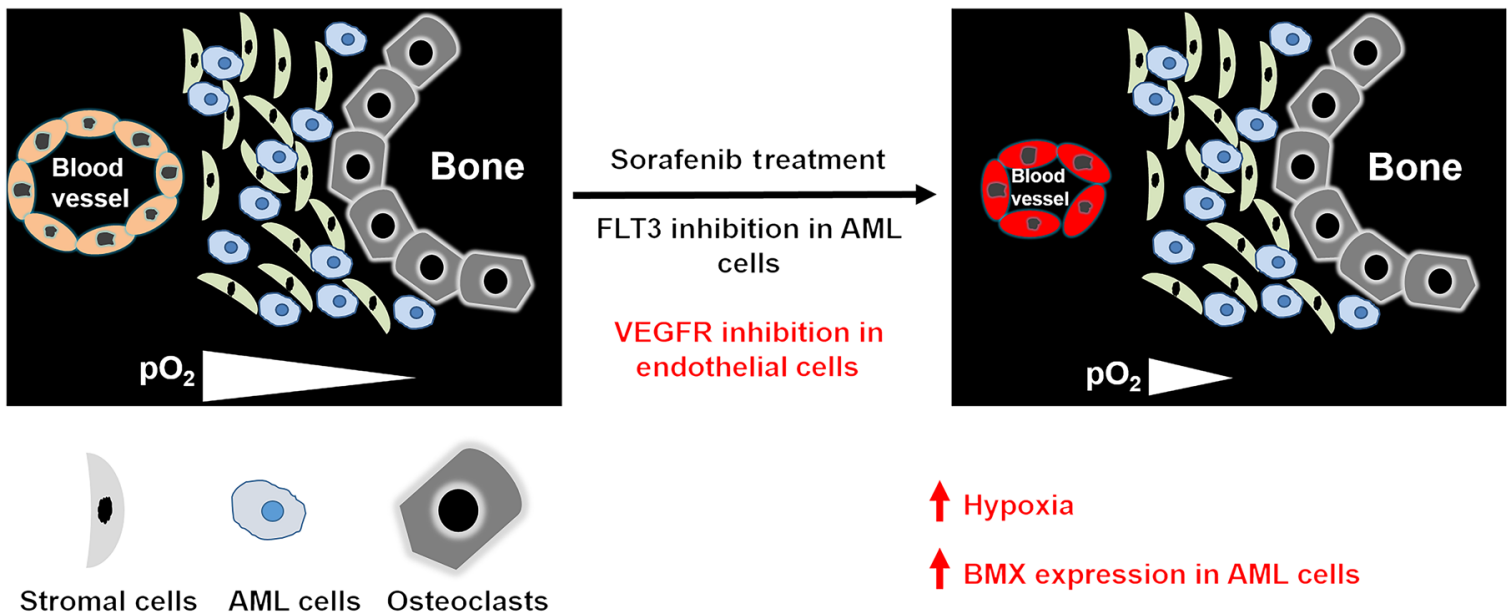

Figure 6. Proposed model. In the bone marrow microenvironment, sorafenib treatment results in FLT3 inhibition in AML cells as well as VEGFR inhibition in endothelial cells. As a result of VEGFR inhibition and antiangiogenic effects of sorafenib, there is increased hypoxia in the bone marrow niche. Hypoxic conditions lead to HIF-dependent BMX upregulation in AML cells, which provides compensatory prosurvival signaling that protects AML cells from deleterious effects of FLT3 inhibition and results in emergence of drug resistance.

started, imaging data showed a clear reduction in tumor load in the mice receiving combination therapy as compared with mice treated with single-agent sorafenib (Figure 5E). To further understand the mechanism responsible for BMX-mediated sorafenib resistance, we examined the effect of BMX activation on several signaling pathways implicated in FLT3 and other prosurvival pathways. We found that overexpression of constitutively active BMX in MOLM13 cells prevented sorafenib-mediated inhibition of phosphorylated STAT5 and AKT (Figure 5F). Conversely, BMX knockdown in MV4-11 cells resulted in increased STAT5 inhibition during sorafenib treatment (Figure 5G). Moreover, ibrutinib treatment phenocopied the effects seen with BMX knockdown (Supplemental Figure 11). These results indicate that BMX activation during sorafenib treatment provides a STAT5-dependent compensatory signaling mechanism rendering the cells resistant to sorafenib-induced growth inhibition.

\section{Discussion}

Adaptive drug resistance is a common cause of treatment failure with both cytotoxic and targeted chemotherapeutics (2). Treatment-induced alterations and/or selection of cancer cells with reduced drug uptake, target gene mutations, and changes in prosurvival as well as cell death pathways are widely recognized mechanisms of adaptive resistance (2). Our study illustrates an alternative mechanism, where therapy-induced changes in the tumor microenvironment contribute to the emergence of therapeutic resistance in AML. We propose (Figure 6) that inhibition of multiple targets by multikinase inhibitors, such as sorafenib, contributes not only to therapeutic efficacy and toxicities, but can also contribute to drug resistance by altering the tumor microenvironment. These findings in AML, along with recent studies in solid tumors (23), highlight the crucial role of treatment-induced changes in microenvironment as an important mechanism of acquired drug resistance.

The components of the bone marrow niche, including stromal and endothelial cells, profoundly influence AML cell survival and drug susceptibility (17). However, as compared with solid tumors, the role of bone marrow angiogenesis in hematologic neoplasms is relatively underappreciated. Studies have demonstrated that there is increased bone marrow angiogenesis in AML patients (24). Our study suggests that treatment-induced alterations in bone marrow angiogenesis induce resistance to other chemotherapeutics, specifically TKIs, through a hypoxia-dependent mechanism. We identified the Tec kinase BMX as a novel hypoxia-dependent gene in AML cells. Hypoxia-dependent BMX upregulation in AML cells was found in both in vitro (1\% hypoxia) and in vivo xenograft models (sorafenib and anti-VEGF antibody treatment). Importantly, hypoxia-dependent BMX upregulation was also observed in other non-AML cell lines like HEK293 cells as well as renal cell carcinoma cells. These studies reveal a hypoxia-dependent transcriptional mechanism of BMX upregulation, which might contribute to BMX upregulation in solid tumors as well, where it is already known to cause resistance to a multitude of anticancer drugs (2527). BMX upregulation provides alternative survival mechanisms through activation of the STAT5 signaling pathway $(25,28)$, contributing to drug resistance. Indeed, we observed that BMX activation can maintain prosurvival signaling pathways (STAT5 and AKT) during FLT3 inhibition in AML cells, likely providing a compensatory mechanism of cell survival and drug resistance.

Tumor heterogeneity is now recognized as an important determinant of therapeutic success as well as the emergence of resistance (29). Cancer therapeutics exert a strong selection pressure that shapes tumor evolution through various mechanisms that are not completely understood (10). This tumor heterogeneity is dependent not only on genetic variations but also on spatiotemporal dynamics of signal transduction networks in different cells (10). Here we find that BMX upregulation might be part of such a compensatory signal transduction network that contributes to sorafenib resistance. Importantly, the FLT3-ITD $^{+}$patient samples that showed BMX upregulation also had FLT3 TKD mutations (Figure 1). It is presently unclear whether the BMX upregulation and emergence of TKD mutations are linked. However, our experiments in in vitro and in vivo models indicate that BMX upregulation may be 
independent of FLT3 mutation status in some TKI-resistance scenarios. First, BMX upregulation in Baf3 cells occurred regardless of the presence or absence of FLT3-ITD or TKD mutations (Figure 3D). Second, in vivo, TKD mutations developed in some mice treated with crenolanib, but BMX was not upregulated, whereas sorafenib treatment resulted in the emergence of TKD mutations and BMX upregulation, as in the patient samples. Future studies are required to conclusively determine the interaction between FLT3 and BMX proteins as well as to delineate the relative role of FLT3 mutations and BMX upregulation in sorafenib resistance.

Collectively, these studies provide several new insights. First, we have identified BMX as a hypoxia-dependent resistance protein. Second, our study highlights the critical role of angiogenesis and bone marrow microenvironment in the therapeutic response in AML. And third, our findings reveal the underappreciated role of "off-target" inhibition in the tumor microenvironment by targeted therapeutics and development of acquired drug resistance.

\section{Methods}

Patients. Four children with relapsed FLT3-ITD ${ }^{+}$AML received maintenance therapy with single-agent sorafenib for $14-54$ weeks until subsequent relapse, as previously described (30). Bone marrow samples were obtained at initial relapse of AML before cessation of sorafenib treatment and at the development of TKI resistance for genomic analysis. Samples were enriched for leukemic blasts by Ficoll purification.

RNA-Seq analysis. RNA sequencing libraries for each tumor sample were prepared with approximately $1 \mu \mathrm{g}$ total RNA using the Illumina TruSeq RNA Sample Prep v2 Kit per the manufacturer's instructions. Sequencing was completed on the Illumina HiSeq 2000 using TruSeq SBS v3 reagents. Illumina paired-end reads were trimmed of adapter using Cutadapt, and any reads less than 30 bp were excluded from downstream analysis. The resulting paired-end reads were aligned to human NCBI Build 37 reference sequence using Burrows-Wheeler Aligner (BWA 0.5.5). FASTQ sequences were mapped to the hg19 genome by STRONGARM, a pipeline that employs STAR, TopHat2, and other mappers. Mapped reads were counted with HTSEQ coverage files, and gene-level FPKM (fragments per kilobase of transcript per million mapped reads) values were then computed and data visualized using Integrative Genomics Viewer. Exon junction data were extracted through the St. Jude Children's Research Hospital in-house RNApeg pipeline. For gene expression analysis comparisons, we obtained counts of the number of reads per gene and FPKM normalization. The data discussed in this publication have been deposited in the NCBI's Gene Expression Omnibus and are accessible through GEO Series accession number GSE104594 (https://www.ncbi.nlm. nih.gov/geo/query/acc.cgi?acc=GSE104594).

Statistical analysis of RNA-Seq expression data. For each sample, we determined the number of reads mapping to each of 935,007 exons and each of 345,970 junctions as well as total reads for each sample. We computed the overall read-count proportion as the number of all reads obtained for the TKI resistance sample (R2) divided by the sum of the number of reads obtained for both the TKI resistance sample (R2) and the pre-TKI sample (R1). Then, for each subject, we computed a similar read-count proportion for each exon and each junction as the ratio of the number of reads mapped to the particular feature for the R2 sample to the sum of the number of reads mapped to the feature for both samples. To numerically stabilize these feature-specific read-count proportions, we added 1 to the numerator and added 2 to the denominator. Then, for each subject, we quantified the change in expression of each feature as the $\log _{2}$-ratio of the feature-specific read-count proportion to the overall read-count proportion. Positive (negative) values of the $\log _{2}$-ratio of read-count proportions indicate that expression of the feature was greater (lesser) at TKI resistance than before treatment. For each feature, the average $\log _{2}$-ratio across samples was computed. Features were then ordered according to the absolute value of the average $\log _{2}$-ratio. For each feature, the 1-sample $t$ test was applied to the subjects' $\log _{2}$-ratios to obtain a $P$ value. No adjustments for multiplicity were performed in this exploratory study with a small sample size. These analyses were performed with $\mathrm{R}$ software (www.r-project.org), and the rctrack (31) package was used to capture a comprehensive archive of all information necessary to computationally reproduce the results.

Gene-set differential expression analysis. We computed a log foldchange statistic as a metric of differential expression for each exon. We then computed this same statistic for each possible permutation of the R1 and R2 labels of the pair of samples for each subject. With the limited sample size of 4 patients, there are only $2^{4}=16$ unique permutations of the $\mathrm{R} 1$ and $\mathrm{R} 2$ labels. We then computed a $z$ statistic as the observed fold-change statistic divided by the SD of the 16 fold-change statistics obtained by permutation. For each gene set, a statistic measuring differential expression was defined as the mean of the squared $z$ statistic over all exons annotated to a gene belonging to that gene set. By the symmetry due to squaring, the permutation of R1 and R2 labels yields 8 unique values of the statistic. Thus, the smallest possible $P$ value in this analysis is $1 / 8=0.125$. This analysis was performed for each of 3,462 gene sets with 100 or fewer genes defined in the molecular signatures database of the Broad Institute. Therefore, we used the robust method of FDR estimation for discrete $P$ values (32) to determine whether there was evidence that there are some true discoveries among the gene sets with $P=0.125$. To facilitate scientific interpretation, the ratio of the observed test statistic to the median of the test statistics obtained by permutation was used as a metric of differential expression at the gene-set level. A value of 1 indicates that the differential expression of the gene set is similar to what would be expected by the permutation model of chance. Larger values indicate greater evidence of differential expression.

Exome sequencing and SNP6 array analysis. Exome enrichment of genomic DNA sequencing from each sample was performed using the TruSeq Exome Enrichment Kit (Illumina, FC-121-1008) on an Illumina HiSeq 2000. The paired-end reads were trimmed, filtered against quality (Phred-like Q20 or greater) and length (30 bp or longer), and aligned to a human NCBI build 37 reference sequence (UCSC hg19); genotypes were called at all positions where there were high-quality sequence bases (Phred-like Q25 or greater) at a minimum coverage of 8, using CLC Genomics Workbench v7.5.1 (CLC Bio). Effective coverage of each exon, averaged at $85 \times$, was obtained by summarizing of the coverage depth of aligned bases within each targeted exon region. The copy number change in BMX for all samples was assessed using the Affymetrix Genome-Wide Human SNP Array 6.0. The copy number analysis was performed using the circular binary segmentation method implemented in Partek software (Partek Inc.). Aberration calls were assigned to those segments with at least 5 markers (probes), $P$ value less than 0.05 , and mean $\log _{2}$-ratio greater than 0.15 (gain) or less than -0.25 (loss). 
FLT3-ITD ${ }^{+}$MOLM13 xenograft model. MOLM13 cells $\left(1 \times 10^{6}\right)$ were administered via tail vein injection to 8- to 12-week-old female NOD. Cg-Prkdcscid Il2rgtm1Wjl/SzJ (NSG) mice (The Jackson Laboratory). Cell engraftment was assessed biweekly by noninvasive bioluminescence imaging, as previously described (16). On day 10 after injection of cells, mice were randomized to treatment groups based on signal intensity, and drug treatments were started. Sorafenib for oral administration and crenolanib for i.p. injection were formulated as previously described (16). Ibrutinib for oral administration was dissolved in 1\% DMSO, 30\% PEG-300, 1\% Tween-80 and brought to final concentration with double-distilled $\mathrm{H}_{2} \mathrm{O}$ right before administration. The leukemic burden was monitored biweekly by bioluminescence imaging. Sorafenib was administered at a dose of $60 \mathrm{mg} / \mathrm{kg}$ once daily for 5 or 10 consecutive days, or daily for 5 days per week until leukemic progression. Crenolanib was administered at a dose of $15 \mathrm{mg} / \mathrm{kg}$ twice daily for 5 days per week until leukemic progression, as previously described (16); this dose and schedule was determined to be the maximum tolerated dose in NSG mice. Ibrutinib was administered at a dose of $50 \mathrm{mg} / \mathrm{kg}$ once daily alone or concurrently with sorafenib $60 \mathrm{mg} /$ $\mathrm{kg}$ once daily continuously for up to 14 days. Bevacizumab (obtained from the St. Jude Children's Research Hospital pharmacy) and B204.1.1 (Genentech) for i.p. injections were dissolved in $0.9 \% \mathrm{NaCl}$. MOLM13-bearing NSG mice were treated with either bevacizumab or B20-4.1.1 (5 mg/kg) starting on day 5 after MOLM13 cell injection, with 2 consecutive treatments on day 8 and day 11 .

For all in vivo studies, at specified time points, mice were sacrificed, and bone marrow was harvested to isolate MOLM13 cells for RNA and whole-cell lysates. After bone marrow was collected, red blood cells were lysed (Sigma-Aldrich, R7757), and MOLM13 cells were stained using human CD45-PE antibody (130-091-230), labeled with anti-PE MicroBeads (130-048-801), and separated from mouse bone marrow cells using positive selection on autoMACS (all from Miltenyi Biotec). Processing of MOLM13 cells for RNA and whole-cell lysates is described below.

Sorafenib and ibrutinib pharmacokinetic studies. During the conduct of the efficacy study, pharmacokinetic studies were performed in NSG mice after 10 days sorafenib and 5 days ibrutinib or combination treatment. Eye bleeds were performed 5 minutes after ibrutinib and 1 hour after sorafenib administration (to coincide with maximum plasma concentrations), and drug concentrations were quantitated in plasma samples using validated HPLC-based methods with tandem mass spectrometric detection in the St. Jude Children's Hospital Comprehensive Cancer Center Pharmacokinetics Core.

FLT3 TKD mutation analysis. RNA was isolated from MOLM13 cells obtained from mouse bone marrow using Trizol (Invitrogen). FLT3 tyrosine kinase domain (TKD) mutations were analyzed by deep amplicon sequencing using cDNA as previously described (33). Briefly, libraries were prepared for either exon 17 or 20 using the Nextera XT kit and run on the Illumina HiSeq 2000 using the the Illumina MiSeq Reagent Kit v2 (300 cycles) with 150 paired ends according to manufacturer's protocols. Sequences were aligned using CLC Genomics Workbench 6.

Immunohistochemistry, immunoblotting, and in vitro kinase assays. Hind limbs were fixed, decalcified with Cal-Rite (Thermo Fisher Scientific), and paraffin-embedded. For CD31 immunohistochemistry of bone marrow containing MOLM13 cells, slides were deparaffinized, followed by permeabilization and blocking of endogenous peroxidase activity with $0.03 \% \mathrm{H}_{2} \mathrm{O}_{2}$. Following incubation with the anti-CD31 antibody
(Pharmingen), secondary antibody (Vector), and HRP-DAB (DAKO), the slides were counterstained with hematoxylin. CD31-positive cell density was determined using standard methods. For BMX and HIF2 $\alpha$ immunohistochemistry of bone marrow containing MOLM13 cells, slides were deparaffinized, followed by antigen retrieval with $1 \%$ SDS. The slides were then incubated sequentially with blocking buffer (2\% BSA, $1 \%$ normal goat serum in PBST) and primary and Alexa Fluor-labeled secondary antibodies (Abcam), followed by mounting with Fluoroshield Mounting Medium with DAPI (Abcam).

Whole-cell lysates from cultured cells or MOLM13 cells obtained from mouse bone marrow were made in RIPA buffer supplemented with $1 \%$ SDS. Invitrogen Bis-Tris gradient gels were used for Western blot analysis, followed by detection by ECL reagent (Cell Signaling Technology). Primary antibodies used were FLAG (Cell Signaling Technology, 14793), BMX (BD Biosciences, 610793), GAPDH (Santa Cruz Biotechnology, sc-20357), BTK (Cell Signaling Technology, 8547), Phospho-BTK (Cell Signaling Technology, 5082), Tec (Cell Signaling Technology, 4987), Itk (Cell Signaling Technology, 2380), $\beta$-actin (Santa Cruz Biotechnology, sc-47778), HIF1 $\alpha$ (R\&D Systems, MAB1536), and HIF2 $\alpha$ (R\&D Systems, AF2997); and secondary antibodies were from Jackson ImmunoResearch.

For in vitro kinase assays, cells derived from mice treated with either vehicle or sorafenib were extracted with a buffer containing $150 \mathrm{mM}$ NaCl, 1 mM EDTA, 1 mM EGTA, 1\% (vol/vol) Triton X-100, 2.5 mM sodium pyrophosphate, $1 \mathrm{mM} \beta$-glycerol phosphate, $1 \mathrm{mM} \mathrm{Na}_{3} \mathrm{VO}_{4}, 10$ $\mu \mathrm{g} / \mathrm{ml}$ leupeptin, $10 \mu \mathrm{g} / \mathrm{ml}$ aprotinin, $1 \mathrm{mM}$ phenylmethylsulfonyl fluoride, $50 \mathrm{mM} \mathrm{NaF}, 0.2 \%$ (wt/vol) dodecyl $\beta$-D-maltoside, and $20 \mathrm{mM}$ Tris ( $\mathrm{pH}$ 7.5). The soluble extracts were subjected to immunoprecipitation with BMX antibody (BD Biosciences, 610793) using standard procedures. Briefly, $500 \mu \mathrm{g}$ cell lysates were incubated with $1 \mu \mathrm{g}$ antibody or normal goat serum and $30 \mu$ agarose protein A/G beads (Santa Cruz Biotechnology). Immunoprecipitates were washed and collected by centrifugation. A portion of the precipitates was subjected to gel electrophoresis and immunoblot analysis to confirm the successful immunoprecipitation of BMX protein, and another portion was used for kinase activity assay. For kinase assay, the same amounts of immunoprecipitate were added to a reaction buffer containing $150 \mathrm{mM} \mathrm{NaCl}, 4 \mathrm{mM} \mathrm{MnCl}, 6$ $\mathrm{mM} \mathrm{MgCl}_{2}, 10 \%$ (vol/vol) glycerol, $1 \mathrm{mM} \mathrm{DTT}, 100 \mu \mathrm{M} \mathrm{Na}_{3} \mathrm{VO}_{4}, 50 \mathrm{mM}$ HEPES (pH 7.5), supplemented with $20 \mu \mathrm{M}$ ATP (with ATP, [ $\gamma$-32P]) and $12.5 \mathrm{ng} / \mu \mathrm{l}$ substrate. The reaction was incubated at $30^{\circ} \mathrm{C}$ for 20 minutes. At the end of reaction, samples were analyzed for amount of substrate phosphorylation as a function of BMX kinase activity.

Generation of phospho-BMX (phospho-Tyr-194) antibody. The rabbit anti-phospho-BMX antibody was generated by Covance using the 118day protocol. Peptide surrounding the Tyr-194 region was used for immunization. Western blot and ELISA were used to test the bleeds for antibody production, followed by purification of phospho-Tyr antibody by affinity purification. The specificity of the purified antibody was confirmed using purified BMX protein activated after an in vitro kinase assay. Dot blot assays with nonphosphorylated and phosphorylated BMX peptide surrounding the Tyr-194 region were carried out by standard methods using nitrocellulose membrane followed by incubation with primary antibody (phospho-BMX) and secondary antibody (anti-rabbit) and detection by chemiluminescence methods. Dephosphorylation assays were carried out by incubation of cell lysates with recombinant lambda phosphatase (New England Biolabs, P0753) at $30^{\circ} \mathrm{C}$ for 0-3 hours, followed by Western blot analysis with phospho-BMX and BMX antibodies. 
Cell culture and conditions. HEK293, MV4-11, and MOLM13 cells were obtained from ATCC, Tert-mesenchymal stromal cells (TertMSCs) were obtained from St. Jude Children's Research Hospital, and RCC4 and 786-O cells were obtained from Sigma-Aldrich. HEK293 cells were cultured in DMEM. AML cell lines MV4-11 and MOLM13, Tert-MSC, RCC4, and 786-O were cultured in RPMI 1640. All media were supplemented with $10 \% \mathrm{FBS}$ and grown at $37^{\circ} \mathrm{C}$ in a humidified incubator containing $5 \% \mathrm{CO}_{2}$. Primary AML cells were cultured in RPMI 1640 supplemented with 10\% FBS and 1× Antibiotic-Antimycotic (Thermo Fisher Scientific). For hypoxic conditions cells were cultured in a $37^{\circ} \mathrm{C}$ humidified temperature-controlled incubator (COY Labs) containing $5 \% \mathrm{CO}_{2}, 94 \% \mathrm{~N}_{2}$, and $1 \% \mathrm{O}_{2}$ for 24 hours.

Cell viability assays. Lipofectamine LTX (Life Technologies) was used for transient transfections, followed by survival assays 24 hours later. For cell line or ex vivo cell viability assays, cells were cultured in either normoxia or hypoxia for 24 hours prior to treatment and dosed for 48 hours, and cell viability was determined by MTT for cell lines or CellTiter Glo (Promega) for primary cells.

RNA isolation, cDNA generation, and reverse transcriptase PCR. RNA was isolated using standard Trizol-chloroform extraction. cDNA was generated from $0.5 \mu \mathrm{g}$ of RNA using the SuperScript III FirstStrand Synthesis System (Thermo Fisher Scientific). Real-time reverse transcriptase PCR (RT-PCR) was performed on BMX and GAPDH using TaqMan probes predesigned by Thermo Fisher Scientific using TaqMan Fast reagents (Thermo Fisher Scientific).

Site-directed mutagenesis and RNAi experiments. Flag-tagged BMX plasmid (RC202002) was purchased from Origene and used for transient transfections in HEK293 cells. The BMX ORF from RC202002 was sub-cloned into pLenti-C-GFP vector (PS100065) and used for stable transduction in MOLM13 cells. Site-directed mutagenesis was carried out in both the Flag and GFP-tagged BMX vectors using the QuikChange II XL Site-Directed Mutagenesis Kit (Agilent) to generate kinase-dead, catalytically active, catalytically active-kinase dead and dominant negative BMX constructs. Mutagenesis primers were designed using the QuikChange Primer Design program and synthesized by Integrated DNA Technologies (IDT). BMX shRNA lentiviral GFP vectors (TL320275) were purchased from Origene, which included scrambled shRNA, shRNA-B, and shRNA-D. Lentiviral production was carried out using the Lenti-vpak Packaging Kit (Origene) and Lenti-X 293T cells (Clontech). Viral supernatant was concentrated and titered using Lenti-X RT-qPCR titration kit (Clontech). Additional shRNA lentiviral particles were purchased from Vigene Biosciences. MV4-11 and MOLM-13 AML cells were plated onto retronectin-coated 6-well plates at $5 \times 10^{5}$ per well and, after overnight incubation, supplemented with $10 \mu \mathrm{g} / \mathrm{ml}$ DEAE dextran, infected with lentivirus at an MOI ranging from 100 to 200, and cultured for 24 hours. Cells were then washed twice and cultured for an additional 24 hours, removed from retronectin, and flow-sorted for $\mathrm{GFP}^{+}$cells. For siRNA experiments in HEK293 cells, briefly, cells were plated in 96-well plates and reverse-transfected with $25 \mathrm{nM}$ siRNA (Sigma-Aldrich and Dharmacon) using Lipofectamine RNAiMAX reagent (Life Technologies). At 48 hours after transfection, cells were transferred to either normoxic or hypoxic (1\% oxygen) conditions for 24 hours followed by whole-cell lysate preparation and Western blot analysis.

Hypoxia reporter element assay. HEK293 cells $\left(5 \times 10^{3}\right)$ were plated overnight on white poly-L-lysine-coated 96-well plates. Cells were transfected with either BMX promoter construct (Switchgear Genom- ics, S701154) or empty promoter construct (Switchgear Genomics, S790005) at $30 \mathrm{ng}$ in combination with the Cypridina TK control construct (Switchgear Genomics, SN0322S) at $1 \mathrm{ng}$, according to the manufacturer's protocol (Switchgear Genomics, Lightswitch Dual Assay kit, DA010). The promoter and empty vector encode a Renilla luminescent reporter gene, called RenSP, while the transfection and normalization vector encodes a Cypridina luciferase. The Renilla luciferase activity obtained under various conditions was normalized with the Cypridina luciferase activity. The data were analyzed by determination of the fold change in comparison with the WT HRE promoter normoxia group.

Chromatin immunoprecipitation-qPCR. Chromatin immunoprecipitation (ChIP) assays were performed using the Pierce Magnetic ChIP Kit according to the manufacturer's instructions. Briefly, cells were crosslinked in $1 \%$ formaldehyde, followed by quenching with glycine, cell harvesting, sonication, and lysate preparation. Lysates were precleared for 1 hour with Protein A+G magnetic beads (EMD Millipore). Precleared lysates were then incubated with $5 \mu \mathrm{g}$ of primary antibody (R\&D Systems) overnight at $4^{\circ} \mathrm{C}$, followed by addition of Protein $\mathrm{A}+\mathrm{G}$ magnetic beads and incubation for at least 2 hours at $4^{\circ} \mathrm{C}$. Finally, the beads were repeatedly washed, followed by elution of the protein-DNA complexes, reversal of cross-links, and DNA purification by phenol/chloroform extraction followed by sodium acetate/ethanol precipitation.

Purified DNA was then subjected to qPCR (ChIP-qPCR) using primers near the putative HRE binding site in the BMX promoter, and percentage input was calculated and normalized as a fold change from IgG.

Statistics. Data illustrated with error bars are the mean \pm SEM. GraphPad Prism (GraphPad Software) was used for statistical analyses. Kaplan-Meier analysis of animal survival was determined by logrank test. All other statistical tests performed were unpaired 2-sided $t$ test with Welch's correction. For all analyses, a $P$ value less than 0.05 was considered statistically significant.

Study approval. All treatments and assessments were approved by the St. Jude Children's Research Hospital institutional review board (Memphis, Tennessee, USA), and informed consent was obtained from all patients or their legal guardians. All animal studies were approved by the Animal Care and Use Committee at St. Jude Children's Research Hospital.

\section{Author contributions}

JGVO, NP, and SDB conceived and designed the study. JGVO, NP, DRB, LL, SAS, and LJJ developed the methodology. CDD, AV, JER, and $\mathrm{HI}$ acquired data, provided animals, acquired and managed patients, and provided facilities. LS, YDW, DF, and SP analyzed and interpreted data, including statistical analysis, biostatistics, and computational analysis. JGVO, NP, and SDB wrote, reviewed, and/or revised the manuscript. SDB supervised the study.

\section{Acknowledgments}

We thank the members of the Baker and Sparreboom laboratories for assistance with experiments and helpful comments during manuscript preparation. This study was supported by the American Lebanese Syrian Associated Charities, NIH Cancer Center Support Grant P30 CA021765, and R01 CA138744 (to SDB). This study was also supported by funds from the Ohio State University Comprehensive Cancer Center, Pelotonia foundation, and NIH Cancer Center Support Grant P3O 
CA016058. NP is supported by a Scientist Development Grant from the American Heart Association. The results shown are in part based on data generated by the TCGA Research Network (http://cancergenome.nih.gov/).
Address correspondence to: Sharyn D. Baker, Division of Pharmaceutics, College of Pharmacy and Comprehensive Cancer Center, The Ohio State University, 500 W. 12th Avenue, Columbus, Ohio 43210, USA. Phone: 614.685.6016; E-mail: baker.2480@osu.edu.
1. Bild AH, et al. Oncogenic pathway signatures in human cancers as a guide to targeted therapies. Nature. 2006;439(7074):353-357.

2. Holohan C, Van Schaeybroeck S, Longley DB, Johnston PG. Cancer drug resistance: an evolving paradigm. Nat Rev Cancer. 2013;13(10):714-726.

3. Döhner H, Weisdorf DJ, Bloomfield CD. Acute myeloid leukemia. $N$ Engl J Med. 2015;373(12):1136-1152.

4. Leung AY, Man CH, Kwong YL. FLT3 inhibition: a moving and evolving target in acute myeloid leukaemia. Leukemia. 2013;27(2):260-268.

5. Daver N, et al. Secondary mutations as mediators of resistance to targeted therapy in leukemia. Blood. 2015;125(21):3236-3245.

6. Davis MI, et al. Comprehensive analysis of kinase inhibitor selectivity. Nat Biotechnol. 2011;29(11):1046-1051.

7. Dar AC, Das TK, Shokat KM, Cagan RL. Chemical genetic discovery of targets and antitargets for cancer polypharmacology. Nature. 2012;486(7401):80-84.

8. Zhang W, et al. Mutant FLT3: a direct target of sorafenib in acute myelogenous leukemia. J Natl Cancer Inst. 2008;100(3):184-198.

9. Zimmerman EI, et al. Multikinase inhibitors induce cutaneous toxicity through OAT6mediated uptake and MAP3K7-driven cell death. Cancer Res. 2016;76(1):117-126.

10. Kolch W, Halasz M, Granovskaya M, Kholodenko $\mathrm{BN}$. The dynamic control of signal transduction networks in cancer cells. Nat Rev Cancer. 2015;15(9):515-527.

11. Tamagnone L, et al. BMX, a novel nonreceptor tyrosine kinase gene of the BTK/ITK/TEC/TXK family located in chromosome Xp22.2. Oncogene. 1994;9(12):3683-3688.

12. Holopainen T, et al. Endothelial Bmx tyrosine kinase activity is essential for myocardial hypertrophy and remodeling. Proc Natl Acad Sci US A. 2015;112(42):13063-13068.
13. Chen S, et al. Tyrosine kinase BMX phosphorylates phosphotyrosine-primed motif mediating the activation of multiple receptor tyrosine kinases. Sci Signal. 2013;6(277):ra40.

14. Jarboe JS, Dutta S, Velu SE, Willey CD. Mini-review: bmx kinase inhibitors for cancer therapy. Recent Pat Anticancer Drug Discov. 2013;8(3):228-238.

15. Rushworth SA, Murray MY, Zaitseva L, Bowles KM, MacEwan DJ. Identification of Bruton's tyrosine kinase as a therapeutic target in acute myeloid leukemia. Blood. 2014;123(8):1229-1238.

16. Zimmerman EI, et al. Crenolanib is active against models of drug-resistant FLT3-ITDpositive acute myeloid leukemia. Blood. 2013;122(22):3607-3615.

17. Konopleva MY, Jordan CT. Leukemia stem cells and microenvironment: biology and therapeutic targeting. JClin Oncol. 2011;29(5):591-599.

18. He Y, et al. Critical function of Bmx/Etk in ischemia-mediated arteriogenesis and angiogenesis. JClin Invest. 2006;116(9):2344-2355.

19. Zhuang J, et al. The expression and role of tyrosine kinase ETK/BMX in renal cell carcinoma. J Exp Clin Cancer Res. 2014;33:25.

20. Inoue T, et al. Cross-enhancement of ANGPTL4 transcription by HIF1 $\alpha$ and PPAR $\beta / \delta$ is the result of the conformational proximity of two response elements. Genome Biol. 2014;15(4):R63.

21. Liu L, et al. Sorafenib blocks the RAF/MEK/ ERK pathway, inhibits tumor angiogenesis, and induces tumor cell apoptosis in hepatocellular carcinoma model PLC/PRF/5. Cancer Res. 2006;66(24):11851-11858.

22. Kim S, et al. Sorafenib inhibits the angiogenesis and growth of orthotopic anaplastic thyroid carcinoma xenografts in nude mice. Mol Cancer Ther. 2007;6(6):1785-1792.

23. Sun Y, et al. Treatment-induced damage to the tumor microenvironment promotes prostate cancer therapy resistance through WNT16B. Nat Med. 2012;18(9):1359-1368.
24. Hussong JW, Rodgers GM, Shami PJ. Evidence of increased angiogenesis in patients with acute myeloid leukemia. Blood. 2000;95(1):309-313.

25. Guryanova OA, et al. Nonreceptor tyrosine kinase BMX maintains self-renewal and tumorigenic potential of glioblastoma stem cells by activating STAT3. Cancer Cell. 2011;19(4):498-511.

26. Dai B, et al. Compensatory upregulation of tyrosine kinase Etk/BMX in response to androgen deprivation promotes castration-resistant growth of prostate cancer cells. Cancer Res. 2010;70(13):5587-5596.

27. Fox JL, Storey A. BMX negatively regulates BAK function, thereby increasing apoptotic resistance to chemotherapeutic drugs. Cancer Res. 2015;75(7):1345-1355.

28. Saharinen P, Ekman N, Sarvas K, Parker P, Alitalo $\mathrm{K}$, Silvennoinen $\mathrm{O}$. The Bmx tyrosine kinase induces activation of the Stat signaling pathway, which is specifically inhibited by protein kinase Cdelta. Blood. 1997;90(11):4341-4353.

29. Almendro V, et al. Inference of tumor evolution during chemotherapy by computational modeling and in situ analysis of genetic and phenotypic cellular diversity. Cell Rep. 2014;6(3):514-527.

30. Baker SD, et al. Emergence of polyclonal FLT3 tyrosine kinase domain mutations during sequential therapy with sorafenib and sunitinib in FLT3-ITD-positive acute myeloid leukemia. Clin Cancer Res. 2013;19(20):5758-5768.

31. Liu Z, Pounds S. An R package that automatically collects and archives details for reproducible computing. BMC Bioinformatics. 2014;15:138.

32. Pounds S, Cheng C. Robust estimation of the false discovery rate. Bioinformatics. 2006;22(16):1979-1987.

33. Drenberg CD, et al. Transcriptome profiling of patient derived xenograft models established from pediatric acute myeloid leukemia patients confirm maintenance of FLT3-ITD mutation. Leuk Lymphoma. 2017;58(1):247-250. 